Erratum

\title{
Cloning of Photoreactivation Repair Gene and Excision Repair Gene of the Yeast Saccharomyces cerevisiae
}

\author{
${\text { Akira } \text { Yasui }^{1} \text { and Marie-Renee Chevallier }}^{2}$ \\ 1 Institut für Biophysik, Freie Universität Berlin, Thielallee 63-67, D-1000 Berlin 33 \\ 2 Laboratoire de Genetique Physiologique, Institut de Biologie, Moleculaire et Cellulaire du Centre National de la Recherche \\ Scientifique, 67084 Strasbourg Cedex, France
}

Due to an unfortunate error, Dr. Akira Yasui's address was changed. The correct address for correspondence and offprint requests is as printed above. 